\title{
Neural Efficiency in Athletes: A Systematic Review
}

\author{
Longxi $\mathrm{Li}^{*}$ and Daniel M. Smith* \\ Department of Physical Education and Health Education, Springfield College, Springfield, MA, United States
}

According to the neural efficiency hypothesis (NEH), professionals have more effective cortical functions in cognitive tasks. This study is focusing on providing a systematic review of sport-related $\mathrm{NEH}$ studies with functional neuroimaging or brain stimulation while performing a sport-specific task, with the aim to answer the question: How does long-term specialized training change an athlete's brain and improve efficiency? A total of 28 studies ( $N=829$, Experimental Group $n=430$ ) from 2001 to 2020 (Median $=2014$, $S D=5.43$ ) were analyzed and results were organized into four different sections: expertnovice samples, perceptual-cognitive tasks and neuroimaging technologies, efficiency paradox, and the cluster analysis. Researchers examined a wide range of sport-specific videos and multiple object tracking (MOT) specific to 18 different sports and utilized blood oxygenation-level dependent (BOLD) functional magnetic resonance imaging (fMRI), functional near-infrared spectroscopy (fNIRS), and electroencephalogram (EEG). Expert-novice comparisons were often adopted into investigations about the variations in general about optimal-controlled performance, neurophysiology, and behavioral brain research. Experts tended to perform at faster speeds, more accurate motor behavior, and with greater efficiency than novices. Experts report lower activity levels in the sensory and motor cortex with less energy expenditure, experts will possibly be more productive. These findings generally supported the NEH across the studies reviewed. However, an efficiency paradox and proficient brain functioning were revealed as the complementary hypothesis of the $\mathrm{NEH}$. The discussion concentrates on strengths and key limitations. The conclusion highlights additional concerns and recommendations for prospective researchers aiming to investigate a broader range of populations and sports.

Keywords: neural efficiency, athletes, sports, neuroimaging, neuroscience

\section{INTRODUCTION}

"As increasing levels of expertise are attained, there are measurable changes in neural activation" (Vickers and Williams, 2017, p. 5). Historically, the neural efficiency hypothesis (NEH) "was first proposed by Haier et al. (1988), who adopted positron emission tomography (PET) to determine the relationship between task performance and level of neural activation during the performance of intelligence tests" (Vickers and Williams, 2017, p. 5). Haier et al. found "an inverse relationship between brain glucose metabolism levels and the score obtained on the intelligence test" (Vickers and Williams, 2017, p. 5). Participants who "had high intelligence scores consumed less energy than those with lower scores and performed more quickly, leading the authors to suggest that superior intelligence was due to neural circuits that performed at faster speeds and with greater efficiency" (Vickers and Williams, 2017, p. 5). 
In general, neural efficiency consists of better performance during the repetition of a task (Babiloni et al., 2009), lower energy consumption in completing same performance (Zhang et al., 2019), and relatively less pronounced alpha ERD as a commonly used index of neural efficiency or spatially selective cortical activation (Del Percio et al., 2008; Babiloni et al., 2010). Higher neural efficiency is characterized by a bidirectional reduction phenomenon encompassing both reduced activation of areas associated with task execution and reduced deactivation of regions associated with irrelevant information processing (Qiu et al., 2019).

In the last decades, several pieces of evidence extended the NEH to the cortical motor and visual systems of "expert athletes" such as elite kendo and gymnasts (Kita et al., 2001), elite rifle and gun shooters (Fattapposta et al., 1996; Haufler et al., 2000; Janelle et al., 2000; Loze et al., 2001; Di Russo et al., 2005; Del Percio et al., 2008), and elite karate and fencing athletes (Del Percio et al., 2009a,b). In recent years, progressive research was conducted in the neuroscience field to interpret athletes' performances. Callan and Naito (2014) addressed four neural mechanisms that might primarily contribute to experts' exceeding performance over novices: neural efficiency, cortical expansion, specialized processes, and internal models. These four neural mechanisms are correlated to revealing evidence about the expert and novice athletes' differences and similarities in brain activity. Recently, Filho et al. (2021) suggested complementary neural mechanisms of neural efficiency, neural proficiency, and transient hypertonicity theoretically correlated with optimal performance in experts.

In this way, investigators in the domain of motor learning suggested that "skilled performance was defined by high levels of automaticity, minimum energy expenditure, and reduced movement times" (Schmidt and Lee, 2014; Vickers and Williams, 2017, p. 5). According to Vickers and Williams (2017), "these documented changes have led to a general 'faster-is-better' approach in terms of defining optimal motor behavior, brain function, and assumptions about how athletes should be trained" (p. 5). For instance, athletes are often instructed to "shift their gaze rapidly and accelerate their thought processes and movements to the point of reducing the level of conscious

\footnotetext{
Abbreviations: ACC, anterior cingulate cortex; aIPS, Anterior intraparietal sulcus; BG, Basal ganglia; BOLD, blood-oxygen level-dependent; BP, Bereitschaftspotential; CNV, contingent negative variation; DAN, Dorsal attention network; DLPFC, dorsolateral prefrontal cortex; EG, Experimental Group; EMG, electromyogram; EP, Externally-paced skill; ERD, Eventrelated desynchronization; ERS, Event-related synchronization; FC, Functional connectivity; FEF, Front eye field; gFCD, Global functional connectivity density; IFG, Inferior frontal gyrus; LIMBIC, Limbic area; LPMCd, Lateral premotor cortex dorsal; LRP, Lateralized readiness potential; MD, mean difference; MP, motor potentials; M1, Primary motor cortex; MFC, Middle frontal cortex; MFG, Middle frontal gyrus; MNS, mirror neuron system; MP, Motor potential; MPCRs, Movement-related cortical potentials; MTG, Middle temporal gyrus; n.r., Not reported or not readily usable/applicable for this type of summary table; NE, Neural efficiency; NEH, Neural efficiency hypothesis; NS, negative slope; OCC, Occipital; PMA, Premotor area; RAP, Reafferent positivity; RCT, right centrotemporal regions; RCTs, Random Control Trials; RP, Readiness potential; rs-fMRI, Resting state functional magnetic resonance imaging; RT, Reaction time; SMA, Supplementary motor area; Spatial-BB, spatial condition with baseball batting-specific S-R mapping; SP, Self-paced skill; SPL, Superior parietal lobule; TRPD/TRPI, Task-related power decreases/increases; yrs., years.
}

control of what they are doing" (Shepherd, 2015; Vickers and Williams, 2017, p. 5). However, reducing conscious control did not lead directly to the non-conscious processes. Although robust evidence was found through non-conscious contributions to action control were strong, the non-conscious and conscious action control were still not fully understood (Shepherd, 2015). Moreover, this runs counter to the literature on quiet eye $(\mathrm{QE})$, which calls for the performer to maintain their visual focus and concentration on a specific location during a critical final phase of movement (Vickers, 2016). Formally, QE is defined as "the final fixation or tracking gaze that is located on a specific location or object in the task environment within $3^{\circ}$ of visual angle (or less) for a minimum of $100 \mathrm{~ms}$ " (Vickers, 2016, p. 119). In a comprehensive review of intelligence and the NEH, "Neubauer and Fink (2009) reported 29 studies in support of the hypothesis, while 18 provided mixed support and nine had contradictory results" (Vickers and Williams, 2017, p. 5). According to Neubauer and Fink (2009), a possible reason for the contradictory results is the variability in task difficulty across the studies they reviewed. That is, some studies incorporated tasks that may not have been demanding enough to find support for the NEH.

In addition, Neubauer and Fink (2009) concluded that the neural efficiency was mostly observed for low-to-moderately difficult tasks and in the frontal lobe of the brain. However, for moderate-to-complex tasks, individuals utilized more cortical resources, leading to the result of positive correlations between brain operation and cognitive ability (Gevins and Smith, 2000; Neubauer et al., 2004; Papousek and Schulter, 2004). According to Vickers and Williams (2017), "this view challenges the widespread assumption that if an athlete is able to move quickly, then his or her neural processes must also function as fast or even faster" (p. 6). The purpose of this study was to systematically review self-paced (SP) and externally paced (EP) skills sportrelated $\mathrm{NEH}$ research incorporating sport-related and simple discrimination tasks along with functional neuroimaging or brain stimulation. The framing question for this review was: How does long-term specialized training change athlete's brain and improve efficiency? In this review, "long-term specialized training" is defined as a planned, structured and progressive development of sport-specific skill to achieve better performance and competitive longevity (Granacher and Borde, 2017).

\section{METHOD}

\section{Search and Screening}

The search strategy included the use of the following databases: PubMed, SPORTDiscus, PsycInfo, MEDLINE Complete, Education Resources Information Center (ERIC), Dimensions, and Google Scholar. Keyword combinations and MeSH terms employed in the search are listed in Table 1. Ten additional references were retrieved from the reference lists of the following papers in addition to the database search: Eslinger and Tranel (2005), Nishiguchi et al. (2015), Park et al. (2015), Di Fronso et al. (2016), Fargier et al. (2017), Gourgouvelis et al. (2017), Yamashiro et al. (2018), Hwang et al. (2019), Nakata (2020), and Parr et al. (2020). As for the additional references, Wei and Li (2017) and Wei and Li (2018), which were written in Chinese, 
TABLE 1 | Flowchart of search strategy.

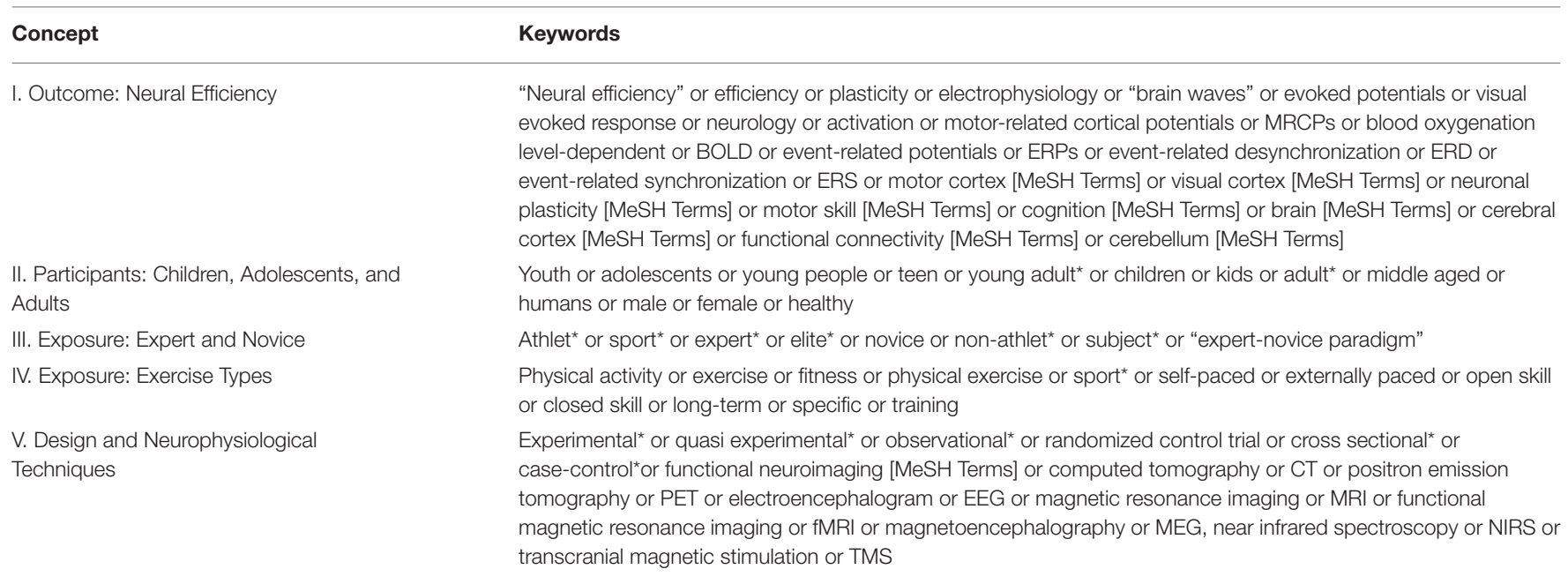

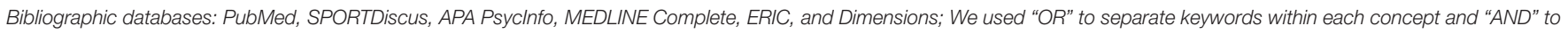

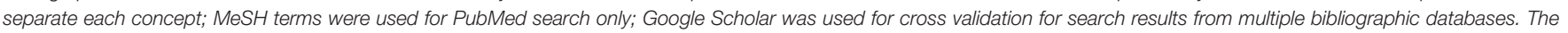

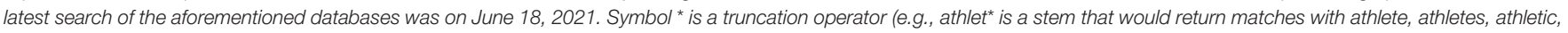
etc.).

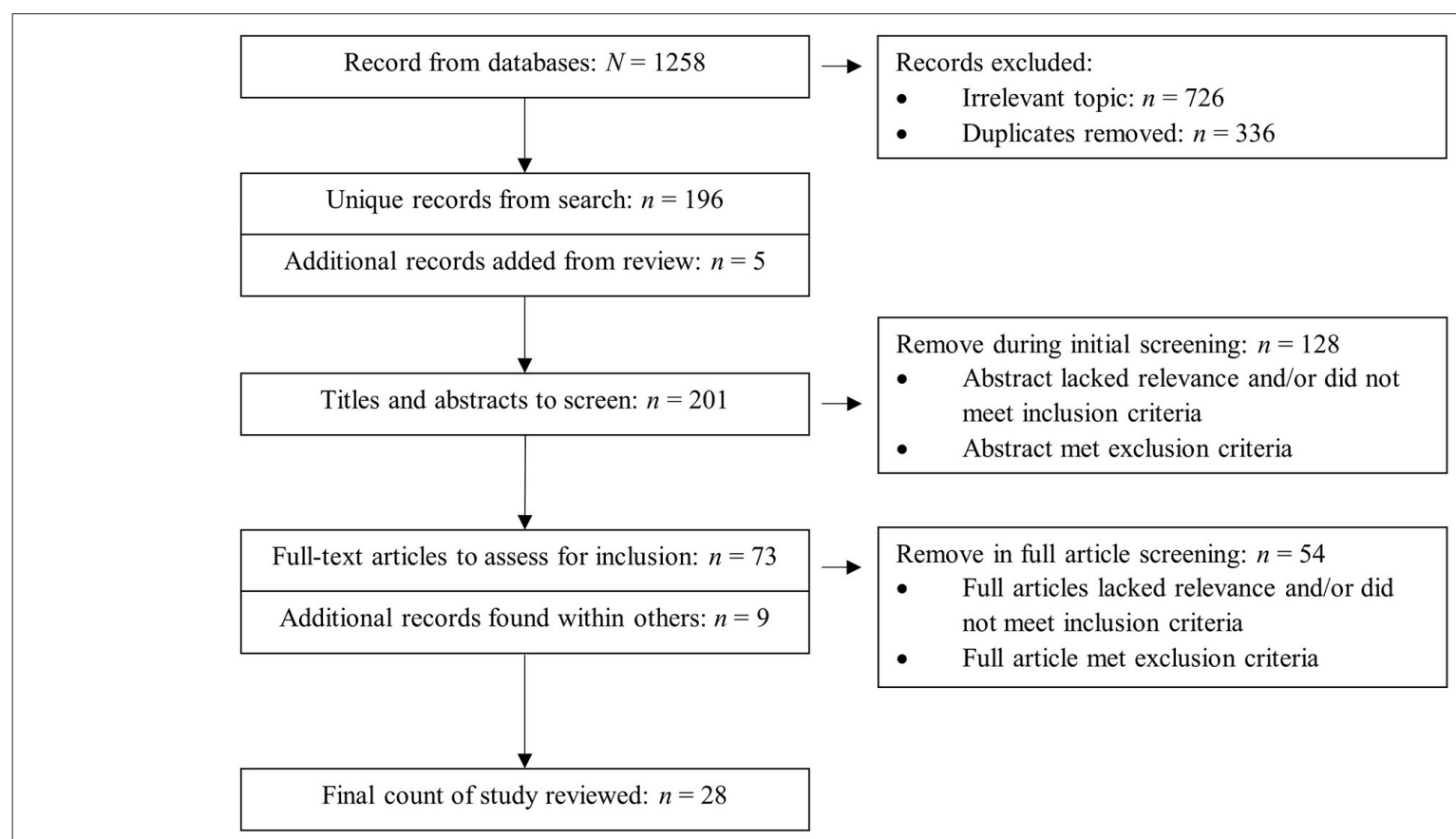

FIGURE 1 | PRISMA flowchart of search strategy and screening.

were found within full-text articles being screened for inclusion. The four-phase PRISMA flow diagram (Figure 1) illustrates the search and screening process.

Inclusion criteria were as follows. First, the study sample included participants under 65 without any cognitive and brain diseases. Second, at least part of the study sample consisted of "experts," defined as subjects highly trained for specific skills (Grabner et al., 2006). That is, within the study sample, at least one group of participants were recognized as having expert domain-specific motor skills (e.g., superior skills as athletes in either individual or group sport, etc.). Both SP skills, which are initiated by the performer (e.g., golf putting, high jumping), and EP skills, in which the timing of performance of the skill is controlled by an outside influence/environment instead of the performer themselves (e.g., football passing, tennis rally), were included (Singer, 2000). Third, the study protocol included each of the following: (1) a resting state eyes-closed and eyesopen recording; (2) a visual stimulus consisting of a domainspecific motor action sequence (e.g., simple discrimination or multiple object tracking task, viewing videos or pictures of 
table tennis serves, rhythmic gymnastics, football actions, etc.) with a general or sport-specific challenge to simulate the stress of high-level competition; and (3) a decision task in which participants reacted to the visual stimulus (e.g., completing a scale to report mental stress, completing a cognitive assessment, evaluating motor performances, matching targets by pressing a number key to indicate the number of probe items, or executing motor movements such as karate punches). Fourth, at least one functional neuroimaging or stimulation technique was used in this research (e.g., PET, fMRI, fNIRS, or EEG) to assess brain regions activation during the tasks. Specifically, activation data including ERPs (amplitude and latency), BOLD signals of cerebral activation, and oxygenated hemoglobin ( $\mathrm{HbO}$ ), deoxygenated hemoglobin ( $\mathrm{HbR})$, and total hemoglobin ( $\mathrm{HbT}$ ) levels between experts and novices. Fifth and finally, the study was an original empirical study written in English or Chinese and published in last two decades (2001-2021). Consequently, studies were excluded if they did not apply expert-novice comparison or outcome excluded cognitive function, motor execution, cortex activation, and functional connectivity (FC), and neither neuroimaging nor stimulation were used metaanalyses or other systematic reviews were excluded.

\section{Data Extraction and Analysis}

Several characteristics were examined for any of the studies that are part of this review: expert-novice samples, visual stimulus tasks, neuroimaging and stimulation techniques, and essential conclusions. The criterion for emphasizing these features was to incorporate standard observations, including brain structures involving, uncover new findings, and recognize methodological strengths and shortcomings, allowing recommendations for future scientific research.

On the basis of similarity and differences within the selected studies, we applied co-authorship and co-occurrence analysis to explore the collaborations among researchers and key concepts via VOSviewer (Version 1.6.16; Van Eck and Waltman, 2010). This procedure was designed to recognize the research collaborations and themes and trends in the field of neural behavior and neuroimaging. According to Van Eck and Waltman (2010), a full counting co-authorship network was constructed based on authors, organizations, and countries and a co-occurrence map was constructed in a three-step process: first, the co-occurrence matrix was used to construct a similarity matrix (see Supplementary Material); second, the VOS mapping technique was used to establish a map from the similarity matrix, which was weighted on total link strength; finally, the map was translated, rotated, and reflected.

\section{RESULTS}

As shown in Table 2, the frequencies of the sports, countries, and neuroimaging types represented across the 28 included studies with reference numbers to refer to the studies hereafter (numbered according to alphabetical order). The median publication year of these studies was 2014 (mean $=2013$; $S D=5.43$; range $=2001-2020)$. These studies $(N=829)$ had
TABLE 2 | Frequencies of expert sports, countries, and neurophysiological techniques in selected studies $(N=28)$.

\begin{tabular}{|c|c|c|}
\hline & $\begin{array}{l}\text { Reference } \\
\text { numbers }\end{array}$ & $\begin{array}{l}\text { No. of } \\
\text { studies }\end{array}$ \\
\hline Karate & $2,3,5-8,11$ & 7 \\
\hline Table tennis & $12,24,25,27$ & 4 \\
\hline Soccer & $9,14,18$ & 3 \\
\hline Basketball & 22,28 & 2 \\
\hline Archery & 15,21 & 2 \\
\hline Badminton & 23,27 & 2 \\
\hline Baseball & 19,26 & 2 \\
\hline Kendo & 13,16 & 2 \\
\hline Football & 4 & 1 \\
\hline Tennis & 27 & 1 \\
\hline Rhythmic Gymnastics & 1 & 1 \\
\hline Gymnastics & 16 & 1 \\
\hline Volleyball & 28 & 1 \\
\hline Fencing & 5 & 1 \\
\hline Swimming & 18 & 1 \\
\hline Shooting & 10 & 1 \\
\hline High Jump & 20 & 1 \\
\hline Golf & 17 & 1 \\
\hline Italy & $1-3,5-10$ & 9 \\
\hline China & $12,22-25,27,28$ & 7 \\
\hline Japan & $\begin{array}{c}13,14,16,18,19, \\
26\end{array}$ & 6 \\
\hline Korea & 15,21 & 2 \\
\hline United States & 4,17 & 2 \\
\hline Turkey & 11 & 1 \\
\hline Sweden & 20 & 1 \\
\hline EEG & $\begin{array}{c}1,2,5-11,13,14 \\
16,19,23-26\end{array}$ & 17 \\
\hline fRMl & $\begin{array}{c}3,4,11,12,15,17 \\
18,20,22,27,28\end{array}$ & 11 \\
\hline fNIRS & 21 & 1 \\
\hline
\end{tabular}

1 = Babiloni et al. (2009); 2 = Babiloni et al. (2010); 3 = Berti et al. (2019); 4= Costanzo et al. (2016); $5=$ Del Percio et al. (2008); $6=$ Del Percio et al. (2009b); $7=$ Del Percio et al. (2010); 8 = Del Percio et al. (2011); 9 = Del Percio et al. (2019); 10 = Di Russo et al. (2005); 11 = Duru and Assem (2018); 12 = Guo et al. (2017); 13 = Hatta et al. (2009); 14 = Iwadate et al. (2005); $15=$ Kim et al. (2014); 16 = Kita et al. (2001); $17=$ Milton et al. (2007); 18 = Naito and Hirose (2014); 19 = Nakamoto and Mori (2008); 20 = Olsson et al. (2008); 21 = Park et al. (2020); 22 = Qiu et al. (2019); 23 = Wang and Tu (2017), $24=$ Wei and Li (2017); 25 = (Wei and Li, 2018); 26 = Yamashiro et al. (2015); 27 = Yang et al. (2020); 28 = Zhang et al. (2019).

fMRI, Functional magnetic resonance imaging; EEG, Electroencephalogram; fNIRS, Functional near-infrared spectroscopy.

Duru and Assem (2018) used both FMRI and EEG in their study; Berti et al. (2019) and Yang et al. (2020) applied rs-fMRl in their study.

a mean total sample size of $29.61(S D=15.14$; range $=7$ $81)$ and a mean expert group sample size of $15.37(S D=8.77$; range $=1-42$ ).

\section{Expert-Novice Samples}

The expert-novice paradigm is commonly used in neurobehavioral research (Callan and Naito, 2014). However, the definition of expertise or experts are inconsistent across 
TABLE 3 | Extraction table of the studies examining the neural efficiency hypothesis in experts-novices paradigm.

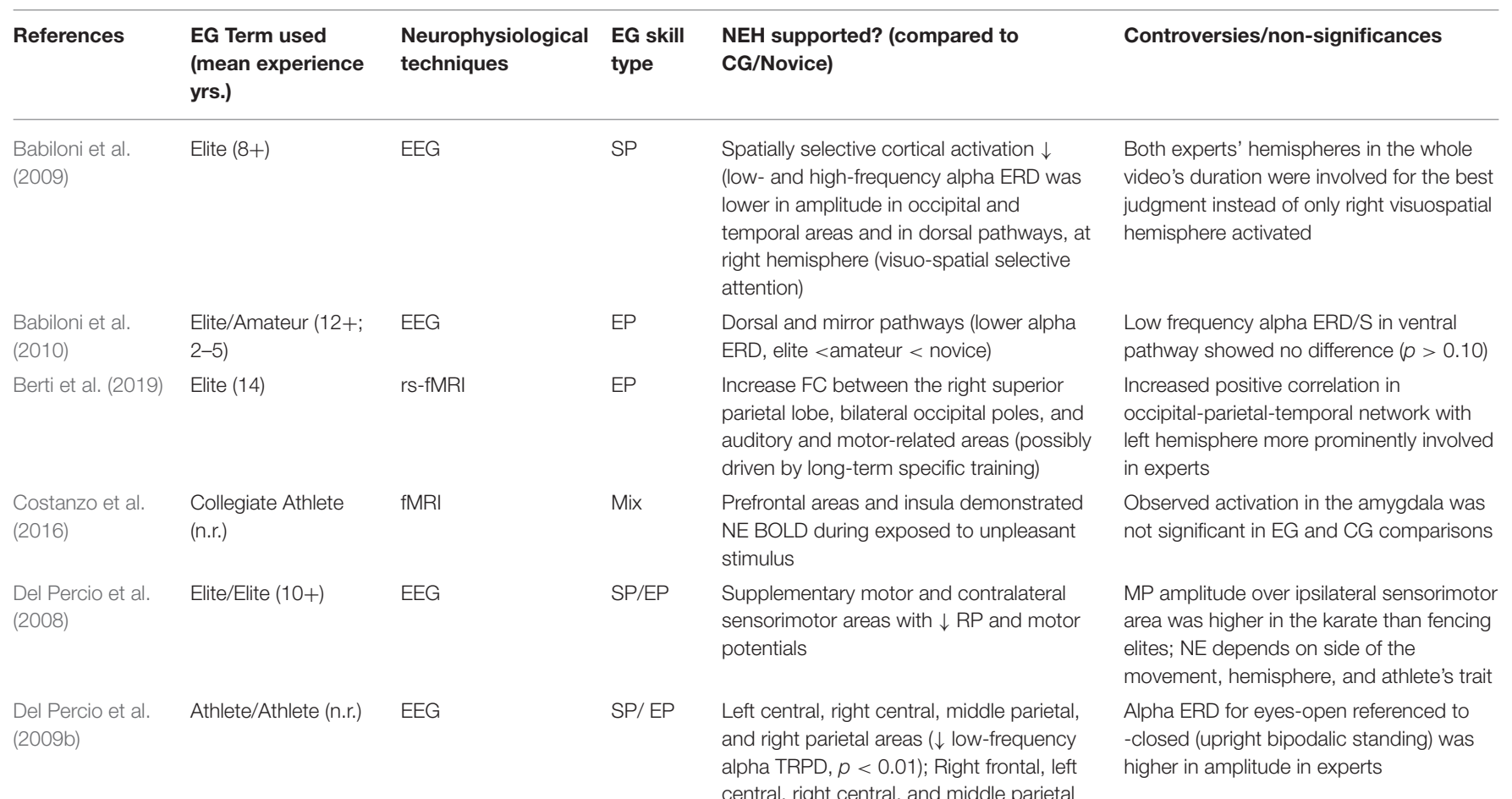

Del Percio et al. Elite Athlete (12+) EEG EP TRPD)
(2010)

Del Percio et al. Athlete (12+) (2011)

Del Percio et al. $\quad$ Player (12.7) (2019)

Di Russo et al. (2005)

Prof

Duru and Assem Elite (7+)

(2018)

Guo et al. (2017)

Athlete (8+)
EEG

EP

EEG

EP

EEG

Mix

Primary motor area, lateral and medial premotor areas, $p s<0.0005-0.005(\downarrow$ high frequency alpha ERD was lower in amplitude in both preparation and execution of the right movements)

Frontal $(p<0.00002)$, central $(p<0.008)$, right occipital $(p<0.02)$ areas $(\downarrow$ low frequency alpha TRPD); frontal $(p<0.00009)$ and central $(p<0.01)$ areas ( $\downarrow$ high-frequency alpha TRPD) n.r.

EP

$\mathrm{BP}$ and $\mathrm{NS}$ 's components related to right finger movements had a later $(p<0.01)$ onset and reduced (BP, $p<0.005$; NS', $p<0.01$ ) amplitude in the left SMA and PMA

$f M R I+E E G$

EP

Frontal (alpha, $p<0.009$; beta, $p<0.029$ ), midline (alpha, $p<0.004$; beta, $p<0.043$ ), parietal occipital (alpha, $p<0.072$; beta, $p<0.081$ ), and RCT regions (alpha, $p<0.0001$ ) $\downarrow$ ERD/S

fMRI Mix
Bilateral middle frontal gyrus; right middle orbitofrontal area, SMA, paracentral lobule, precuneus, angular gyrus; left supramarginal gyrus, inferior temporal gyrus; middle temporal gyrus, bilateral lingual gyrus and left cerebellum crus $\downarrow$
Unclear the reason of $\mathrm{NEH}$ was more represented in right (dominant) than left movements)

Reduction of alpha power for eye-open to close condition (upright bipodalic standing) was greater in experts

A prominent and bilateral parietal alpha ERD was greater $(p<0.05)$ in experts (Low-frequency alpha sub-band: P4, $p=0.01$; high-frequency alpha sub-band: $\mathrm{C} 4, p=0.01, \mathrm{P} 3, p=0.04, \mathrm{P} 4$, $p=0.002$ )

No difference was found between expert and novice for MP and RAP

n.r.

Precuneus showed $\uparrow$ under the sports related vs. unrelated stimulus condition in experts 
TABLE 3 | Continued

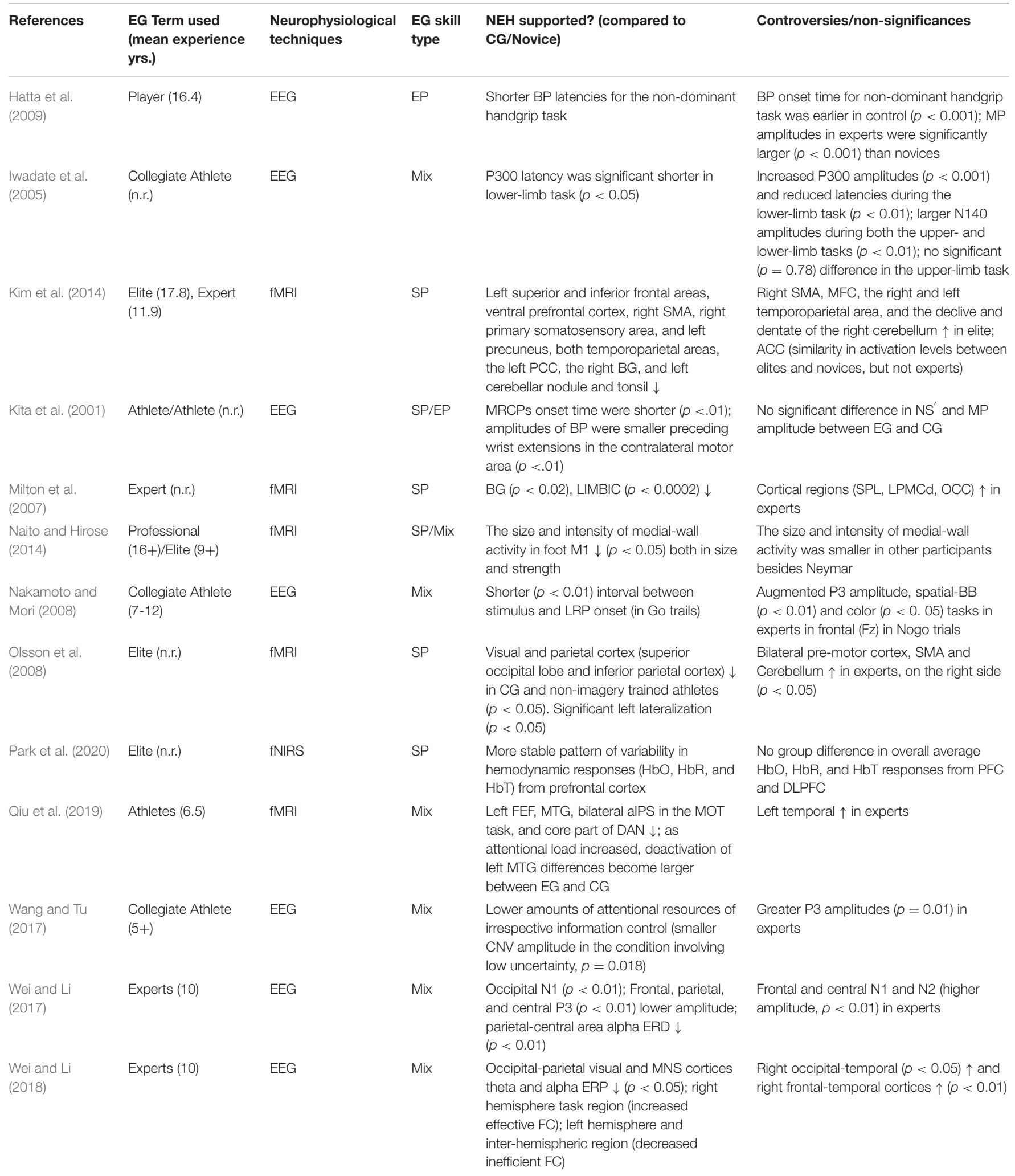


TABLE 3 | Continued

\begin{tabular}{|c|c|c|c|c|c|}
\hline References & $\begin{array}{l}\text { EG Term used } \\
\text { (mean experience } \\
\text { yrs.) }\end{array}$ & $\begin{array}{l}\text { Neurophysiological } \\
\text { techniques }\end{array}$ & $\begin{array}{l}\text { EG skill } \\
\text { type }\end{array}$ & $\begin{array}{l}\text { NEH supported? (compared to } \\
\text { CG/Novice) }\end{array}$ & Controversies/non-significances \\
\hline $\begin{array}{l}\text { Zhang et al. } \\
\text { (2019) }\end{array}$ & Expert (9.8-10.7) & fMRI & Mix & $\begin{array}{l}\text { Left putamen, inferior parietal lobule, SMA, } \\
\text { postcentral gyrus, right insula } \downarrow \text {; better } \\
\text { temporal congruence between motor } \\
\text { executions and motor imagery } \\
(p<0.001) \text {; effective in the representation } \\
\text { and the interoception of the motor } \\
\text { sequences in volleyball }(p<0.001 \text { ) and } \\
\text { basketball }(p<0.01) \text { experts }\end{array}$ & $\begin{array}{l}\text { Experts involved more efficient motor } \\
\text { simulation and less neural effort in } \\
\text { performing the integrated representation } \\
\text { of their self-sport }\end{array}$ \\
\hline
\end{tabular}

$\downarrow=$ decreased activation; $\uparrow=$ increased activation; Mix $=$ The study includes the type of sport appeared both self-paced as well as externally paced characteristics, such as tennis, serving is self-paced but rally is externally paced; SP/EP = The study includes both self-paced as well as externally paced sports in either expert or novice group.

expertise studies (Baker et al., 2015). Given the taxonomy by Baker et al. (2015), novices are defined as individuals in early phrases of skill development with limited skill, and experts are eminent athletes reaching peak levels of skill. Although the standard of identifying experts in selected studies varies, the years of experience and belonging to athletic teams (e.g., national team) are two consistent criteria. Studies 4, 6, 14-16, 19, and 20 did not include years of experience data, but participants in expert groups were either experienced collegiate athletes (4, $14,19)$ or national team members/top-ranking athletes in their countries $(6,15,16,20)$. The original experimental group terms use in selected studies are listed in Table 3. The competitive level of expert groups ranged from professional or elite athletes $(1,2,6,7,10,15,18,20,21)$ to national level, regional, or collegiate athletes $(3,4,5,8,9,11,12-14,16,17,19,22-27)$. Among studies, experts reported the number of years for their participation in the perspective sports, and the mean of these is 11.27 ( $S D=4.96$ ) years. A range of approaches to sampling was apparent across the 28 studies. Noteworthily, participants in study 15 included Olympic gold medallist archers, and study 18 included professional soccer player Neymar. These participants had 17.83 and 16 years of experience in their respective sports.

The most popular approach was to investigate one sample of expert athletes and one sample of participants who have never practiced in the sport before (i.e., non-athlete or novice group), which aligned with studies 1-17, 19-22, 24, 25, and 27. In study 5-7 and 9, age and gender were used as covariates in the subsequent statistical study to rule out the possibility that minor variations in age and gender impacted the final statistical results. Study 2, 5, 6, and 16 explicitly divided participants into three levels of experience (elite, amateur, and athletes in other sports). Similarly, study 2 had three groups (elite, amateur, and novice) consisting of 12 years, 2-5 years, and no experience playing karate, respectively. The novice group $(2,5,7,8)$ did not reach the competitive or amateur level of playing karate or other sports similar to karate (i.e., kung fu, etc.).

Study 23,26 , and 28 took a unique approach in which expert athletes and tasks from two separate sports were recruited, basketball and volleyball, baseball comparing to Track and Field and Swimming, as well as badminton and track and field, respectively. In this way, each group operated in both roles of experts and novices in different sports. Noteworthily, in study 24 and 25, both experts and novice participants $(n=39)$ were selected randomly, 19 national level competitors (Age $=20.2$ \pm 1.2 ) and 20 novice university students (Age $=20.8 \pm 0.9$ ), respectively. In studies $1-10,12-17,20-28$ participants were right-handed and age and gender were equally balanced in expert and non-expert groups.

\section{Perceptual-Cognitive Tasks and Neuroimaging Technologies}

In EEG (1, 2, 5-8, 11, 12 13, 14, 16, 19, 23-26), fMRI (3, 4, $11,12,15,17,18,20,22,27,28)$, and fNIRS (21) studies the subjects were comfortably seated in front of a computer monitor with a distance of $60-95 \mathrm{~cm}$ to the monitor. Subjects watched the domain-specific motor action sequence videos as the visual stimulus. Examples included simple discrimination tasks (24) or MOT task (22), table tennis serves (25), rhythmic gymnastics (1), and football actions (4). After each video stimulus, participants were required to make a decision in response to the visual stimulus. Examples included completing a scale to report mental stress, completing a cognitive assessment, evaluating motor performances, matching targets by pressing a number key (5$7,9,10,12,15,17,18,22,23,27)$, moving a joystick to indicate the number of probe items (4), and executing motor movements such as karate punches. In order to collect behavior data, in 
some studies (e.g., 1, 2), subject's personal coach or similar level expertise completed the same evaluation. The coach's evaluation or other pre-established criteria was used as the gold standard for assessing action judgment in the current participants to analysis the behavioral data (e.g., judgment error). Moreover, tasks also involved decision-making (left vs. right, hit vs. miss, etc.). In fMRI studies $(3,4,11,12,15,17,18,20,22,27,28)$, experimental procedures were similar to EEG studies in that participants were presented with visual stimuli showing sport event-related design videos (similar to the studies reviewed by Smith, 2016). Participants evaluated the performance related to the video and responded by pressing a button (14), directing a joystick (5) or a motor action when the imperative stimulus appeared (4). Across the selected studies, participants observed video clips of rhythmic gymnastics performance $(1,7)$, karate $(5,6)$, motor control in soccer (18), negative sense in football (4), table tennis tasks $(12,18,27)$, fencing attacks (5), archer aims at the target (15), basketball feel throws (22), and badminton serves $(23,27)$. All studies had provided associated criteria with the task.

In this review, we found there to be three distinct advantages of using EEG (17 studies), compared to using fMRI (11 studies) or fNIRS (one study). First, "studies can be carried out in the live 'in situ' setting in sports," such as rhythmic gymnastics, archery, table tennis and fencing $(5,12,24,25,27)$, "thereby allowing the measurement of neural activation as specific sport tasks are performed successfully or unsuccessfully" (Vickers and Williams, 2017, p. 15). Second, EEG studies (1, 2, 5-8 11, 12 13, 14, 16, 19, 23-26) provide "precise measurement of the temporal activation of neural networks as movements are prepared," unlike fMRI ( 3 , $4,11,12,15,17,18,20,22,27,28)$, which lacks the temporal resolution to provide this information (Vickers and Williams, 2017, p. 15). Third, eye movement potentials "can be determined as EEG is recorded" (1, 2, 5, 6-9, 19, 23), "thereby providing insight into the spatial locations of gaze fixations and the duration of focus on critical cues" (Vickers and Williams, 2017, p. 15). Quiet eye also identifies "the critical phase of the movement when the $\mathrm{QE}$ must be focused to lead to successful vs. unsuccessful trials" (Vickers and Williams, 2017, p. 15; Mann et al., 2016). Meanwhile, EEG studies $(23,25)$ that determined theta activation levels in table tennis serve are reviewed, as well as studies (5-7) that have determined the EEG, EOG, and EMG concurrently. In sum, all studies applied at least one neuropsychology technology consisting of fNIRS, fMRI, or EEG (study 11 used both fMRI and EEG) to examine areas of neural activation during eventrelated stimuli controlled for baseline activation, except for study 18 , in which investigators conducted one extra session to record participants' foot movements.

\section{Efficiency Paradox}

We assume neural efficiency shown in athletes is considered as an integration of neuroanatomical structure changes (neural plasticity) and neural proficiency of higher cognitive processing and neural network through long-term training in specific sports. The accumulative evidence supported our assumption (CalvoMerino et al., 2005; Rypma and Prabhakaran, 2009; ZamoraLópez et al., 2010; Paolicelli et al., 2011; Turella et al., 2013; Wolf et al., 2014; Nakata, 2020; Filho et al., 2021). In order to illuminate the mechanisms revealed in previous studies, a schematic overview of neural efficiency in the athletic brain is depicted in Figure 2. We extracted both supporting and conflicting evidence of the NEH and listed the key evidence in Table 3. Among all of the studies (except 9, 13), the revealed data can be theoretically interpreted in terms of neural efficiency in some degree. The summarized studies, in particular, typically report a negative association between brain activation and optimal task performance $(1-8,10-20,22-28)$. This may indicate that experts use their brains more efficiently with less energy consumed (a smaller number of resources are allocated) than novices or non-athletes for performance of a task. To the extent that motor imagery may be seen as a preparation for execution, lateralization effect resulted in left hemispheric specialization in experts during execution of imagery based on established motor representations showed supportive evidence of the NEH $(5,7$, $10,17,20)$, especially in right dominant sports $(24,25)$. Given the broad body of evidence supporting the NEH, we briefly summarize the findings that reported only partial support for the $\mathrm{NEH}$; that is, only for certain categories $(1-8,10,12-28)$, under specific conditions/tasks $(6,12,19,21,24-28)$, for specific brain regions $(1-8,10-28)$, or even presented the opposite finding (1$10,12-28)$. Moreover, the phenomenon of $\mathrm{QE}$ is introduced in the context of the efficiency paradox (Mann et al., 2016). But on the other hand, studies $(1,5-7,9-11,15,22-24,25-28)$ on complex visuospatial, visual search, motion observation, and cognitive tasks have shown that athletes' parietal, central, and other areas have higher cortical activation, inconsistent with the NEH. Possible reasons are as follows. First, the control group lacked specific experience and strategy, less task confidence and effort investment, less related neural cortex resource investment, and lower task-related brain activation. Second, as some authors have noted, it is possible that as activation in the Default Network $(\mathrm{DN})$ increases, the athlete's control processing decreases (CalvoMerino et al., 2005; Turella et al., 2013; Wolf et al., 2014). Third, long-term specific training may lead to the reorganization of cortical activation circuits and induce different cortical circuits. Moreover, considering the conflicting results, the phenomenon of QE was introduced in the context of the "efficiency paradox" (Mann et al., 2016).

Mann et al. (2016) identified an efficiency paradox that runs contrary to the NEH. The endorsement of a "longer is better" recommendation remains simplistic from both a scientific and intuitive standpoint, and the primary mechanisms correlated with this recommendation persist speculatively. However, extensive evidence emanating from previous studies shows that, paradoxically (i.e., the polar opposite), the QE control associated with superior motor skills is slower and of long duration. Even for tasks that are fast and ballistic, like table tennis serve $(12,24,25,27)$, the QE onset is early, on a specific location $(4,14,22,27,26)$, and has a duration that is longer when identifying the opponent's movement than when reacting. Similarly, in soccer, badminton, and archery $(15,27)$, the QE tracking duration is longer on successful than on unsuccessful shots which the expert's cortex activation is greater than novice $(2,5,9,22,26)$. Due to the limited capacity of cognitive capacity of human brains $(3,27)$, the athlete seems to find 


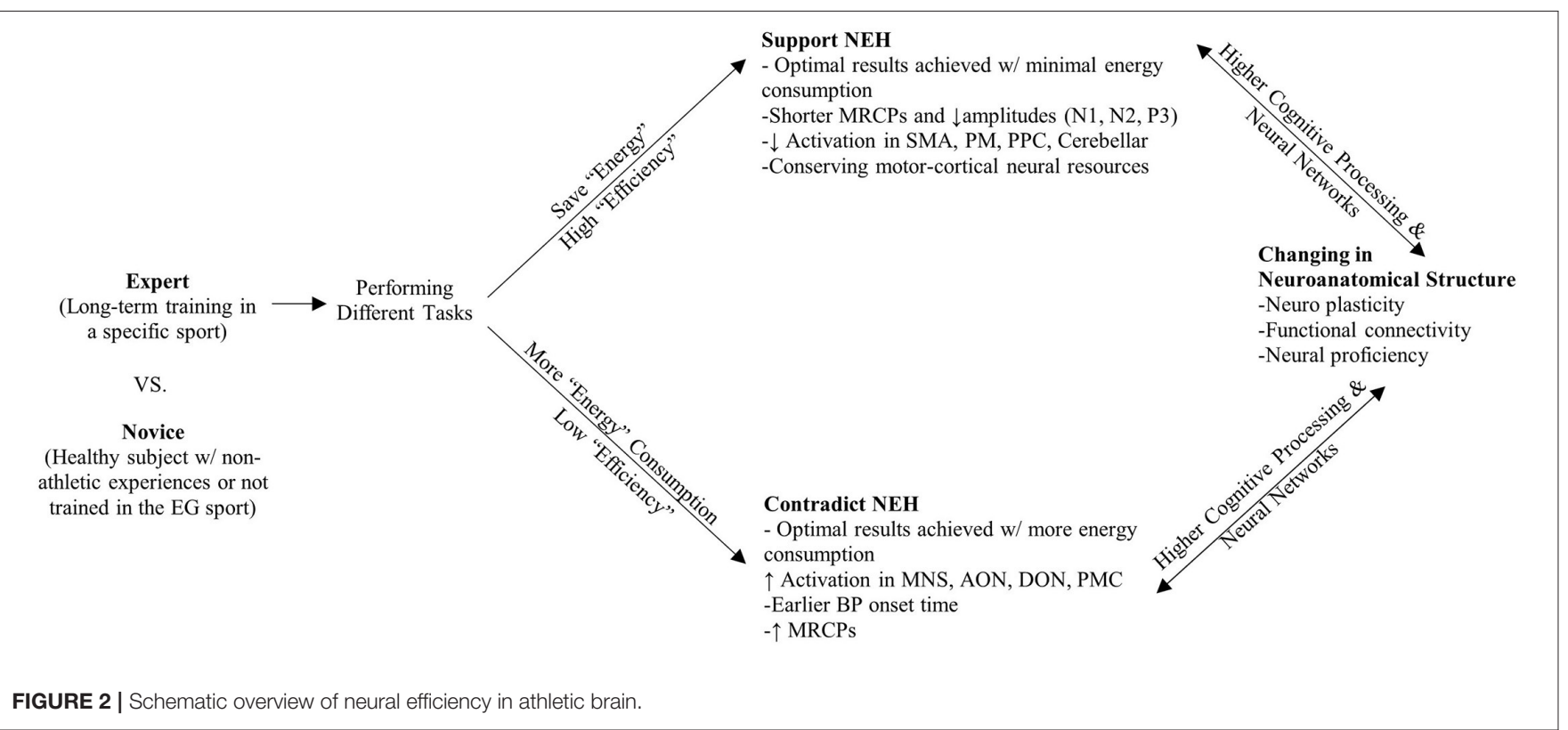

ways to navigate complex spatial information earlier and to maintain their focus under the most challenging of situations. Additionally, "at the highest competition level of sport, athletes are faced with immense levels of pressure, unpredictable playing conditions, and actions of opponents and officials that can be difficult to control" (Vickers and Williams, 2017, p. 9). Thus, the different perspectives related to the NEH need to be understood situationally. There are generally two categories of visual stimulus tasks in selected studies regarding the simplemoderate (e.g., discriminate color, shapes, or remain bi/monopodalic upright standing) and moderate-complex (e.g., identify a backspin serving in table tennis from video clips or react to visual stimulus by executing motor movements) stimulus tasks. Compared to simple-moderate tasks, moderate-complex task involved more sports specific motor skills which possibly modulate high-level cognitive system resources allocating to task demands (Eng et al., 2005; Kliger and Yovel, 2020). In general, experts tended to perform better than novices in both types of visual stimuli tasks (simple-moderate: $6-8,10-14,16$, $17,19,22,24,27$; moderate-complex: 1-5, 15, 18, 20, 21, $23,25,26,28)$ but the activation cortex areas and pathways are inconsistent (see Table 3). These results indicated that the judgment of observed sporting actions is linked to relatively lower levels of alpha ERD, which may be a sign of spatially selective cortical activation or neural efficiency (1-12, 14-28). Specifically, studies 14,22 , and 27 reported a further step in defining brain correlates of the $\mathrm{NEH}$, aligned with Babiloni et al. (2010) which can be considered as a model of continuous plastic train-related adaptation in the aforementioned athletes, and studies $(23,28)$ concluded similarly when performing a task related to an individual's particular sport domain, competence in sports is correlated with proficient control of brain function during cognitive and motor preparation, as well as response execution $(14,21,22)$. In fMRI studies $(3,27)$, specifically in the resting-state condition, there were reduced connections between brain regions (e.g., left IFG and MFG) and remaining brain voxels in experts. This aligns with the $\mathrm{NEH}$ because the reduced connections (i.e., conservation of resources), might reflect improved global efficiency in the athlete's brain. The supporting and contradictory evidence for the NEH is discussed further in the Discussion section.

\section{Results From Co-authorship and Co-occurrence Analysis}

The results of co-authorship and co-occurrence analysis were demonstrated regarding to two visualization maps (Figures $\mathbf{3}$, 4). In both maps, items (authorships and co-occurrence terms) are represented by their labels and circles. The size of the label and circle of an item is determined by the weight of the item (Van Eck and Waltman, 2010). Co-authorships were weighted on total link strength (items $=123$, clusters $=19$, links $=495$, total link strength $=664$, and Maximum iterations $=1,000)$. The demonstration of authorship clusters was verified across the studies. Study 12, 22, and 28 included a similar sample size, findings, and authors working collaboratively in the field. Authors from studies 1, 6, 24, and 25 shared similarities in sample size, imaging method, and task protocol. Authors from studies 5, 9 and 25 are decentralized because those studies concluded contradictory results (as discussed in the previous section). Lastly, clustering may be due to similarities in the imaging method and/or sample size, and/or the close collaboration among different research teams in different countries. By nations, coauthorships reflected that authors from Italy were most active with consistent and recent publications, followed by scholars from Japan, China, Korea, United States, Turkey, and Sweden, respectively (see Figure 3). The co-occurrence map was weighted on total link strength (items $=38$, clusters $=4$, links $=485$, total link strength $=6195$, and Maximum iterations $=1000$ ). The transition of overlay from dark to light demonstrated the trends of published research from 2001 to 2020. The keyword 

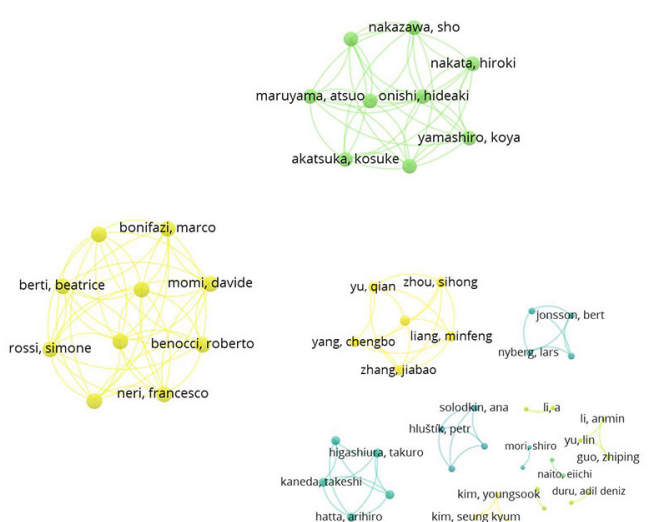
hatra, arihiro kim, seung kyum

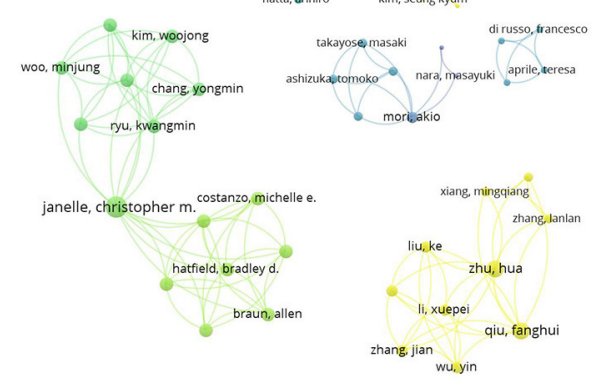

Bas Vosviewer

FIGURE 3 | Co-authorship map.

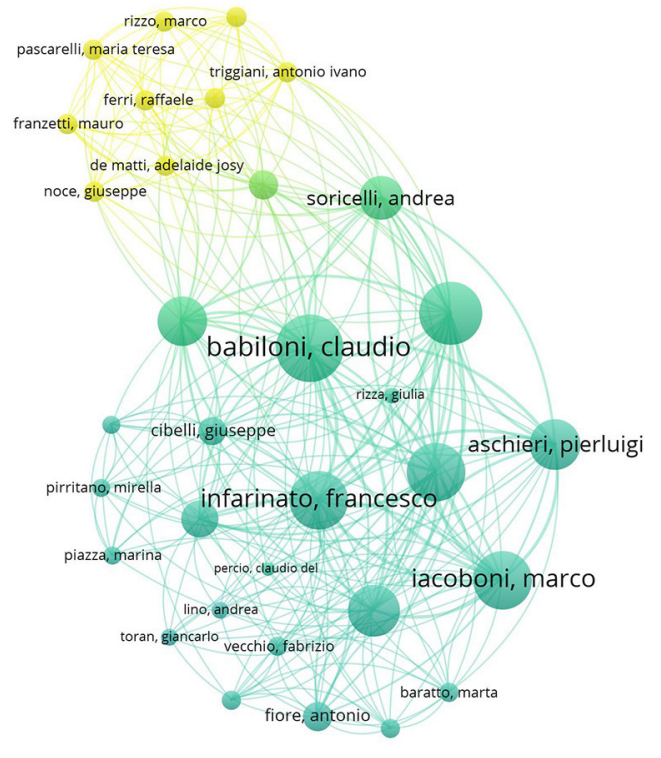

$2000 \quad 2005 \quad 2010 \quad 2015 \quad 2020$

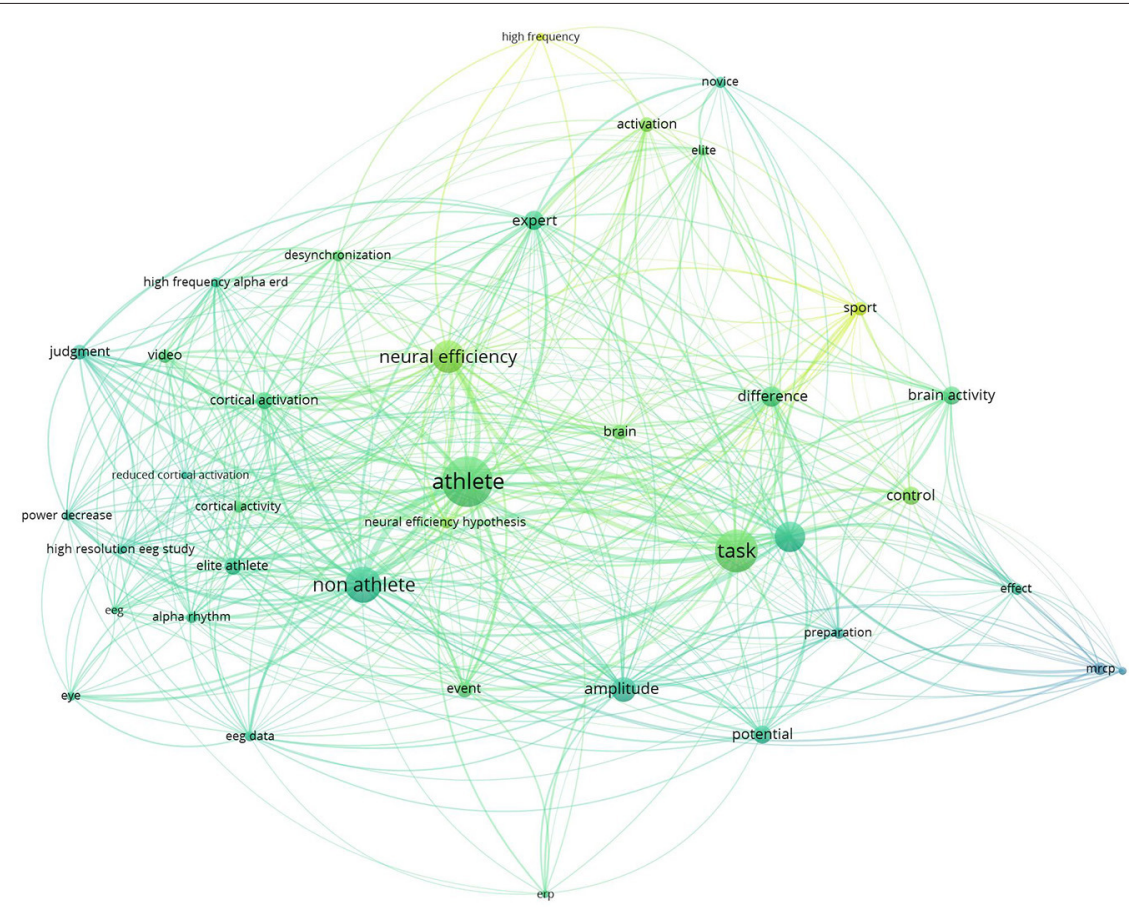

Bi vosviewer

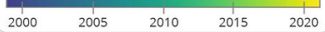

FIGURE 4 | Co-occurrence map and key terms of selected studies. 
and main results from the selected studies were categorized into clusters which demonstrated the core focus on athletes, non-athletes, neural efficiency, and tasks (see Figure 4). The results of co-authorship and co-occurrence analysis provided complimentary bibliography evidence that the expert-novice paradigm and $\mathrm{NEH}$ were popularized in most recent research associated with worldwide collaborations.

\section{DISCUSSION}

The studies included in this review had a number of strengths. First, the sample sizes of the median overall sample sizes and expert group sample sizes were 30 and 15, respectively, which is greater than is often found in functional brain imaging studies (Szucs and Ioannidis, 2020). Studies included sample sizes to achieve adequate statistical power (except for study $12 ; N=7$ ) and ensure balance between the sample sizes of expert and novice study groups. Second, the selected studies consistently took measures to account for many possible between-group confounding variables, such as age, vision, dominant hand, and psychological or neurological conditions. Studies 5, 6, 9,27 , and 28 were particularly strong in reporting possible confounding variables and the steps taken to control for them. Third, all studies also implemented within-participant controls by randomly presenting stimuli and applying multiple trials of each experimental condition.

There were also several limitations. First, in one study, the control group was used to compare cognitive performance while the control subjects did not perform any kinematic task (3). The basic movements of the karate discipline, however, restrict the FC analysis of athletes, and subjects in the control group cannot replicate these gestures (3). It is not reliable to conclude that "differences in FC results are specific for karate athletes, as athletes of other martial arts have not been tested" (Berti et al., 2019, p. 11). Second, the evidence found in the selected studies indicates that expert advantages in perceptual-cognitive tasks mainly occur in sport-specific tasks rather general tasks (1, 3 12, $19,22,24,25)$. Therefore, it is still unclear whether a generic perceptual-cognitive training intervention can be effective to enhance sport-specific skills in athletes (Fleddermann et al., 2019). However, contrary to the NEH, the research on motion observation and representation tasks in dance and basketball shows that compared with novices, the mirror neuron system (MNS) of expert motion understanding is more activated or showing no difference $(1,2)$, which may be related to the moderating effect of task difficulty, DN, and confidence (CalvoMerino et al., 2005; Turella et al., 2013; Wolf et al., 2014). Finally, it should be noted that all selected studies were cross-sectional studies (except study 20, consisting of a 6-week intervention without randomization) based on a comparison among two/three groups of participants. Accordingly, the investigators could not rule out that some differences may have already existed before practicing sports. Thus, more longitudinal studies are needed. In addition, it should be noted that many of the studies included in this review did not report effect sizes or enough information to enable readers to compute effect sizes.

The evidence presented above suggests that experts are more successful than novices when reacting to an upcoming event while recruiting less attentional resources and devoting more attention to subsequent goal analysis in more unexpected situations. This is in keeping with studies 12, 23, 24, 25, and 27, which asserted that players of racket sports demonstrate more attentional flexibility than novices. Despite the evidence that athletes have greater neuro-cognitive processing than novices, findings from study 7 might not be fully explained by the $\mathrm{NEH}$. With respect to the NEH, experts have been shown to have lower cortical resource expenditure (i.e., more automaticity) than novices on several occasions. In study 23, despite evidence that badminton players process the cue more automatically than athletic controls, they seem to put more effort into goal processing. The neural proficiency hypothesis $(\mathrm{NPH})$, a broader term, has been proposed to explore the connection between neural activation and superior performance (Bertollo et al., 2016). According to the NPH, an athlete's effort to sustain a high level of performance by moving proficiently between optimal-automatic and optimal-controlled performance states can modulate brain activity (28), implying complex shifts in the implementation of different strategies to maintain optimal performance (Bertollo et al., 2016). In this way, efficient and effortful processing during performance, the degree of effort as well as the cognitive demands of the task's control systems, can be modulated. Compared to the novices, the neural efficiency in experts (12) not only decreased in activation of the occipital-parietal visual cortex and mirror system cortex, but also increased effective FC and decreased inefficient FC $(25,27)$ in the specific brain region (Cook et al., 2014). In the meantime, athletes can economize attentional resources when processing sport-specific cues in order to respond accurately and quickly. Recently documented meta-analytic EEG evidence in SP sports has suggested that the $\mathrm{NEH}$, transient hypofrontality, and neural proficiency are complementary to each other in optimal motor performance (Filho et al., 2021). Although Filho et al. reported a non-significant increase in alpha and decrease in theta activity in expert-novice paradigm, they argued that theoretically the ERP results are congruent with the NEH (Grabner et al., 2006; Dunst et al., 2014). Compared to the evidence from EP and mixed skill sports, the ERP and BOLD results in studies $(11,12,22,25)$ align with SP sports, and studies $(2-4,13,14,19,23,24,26-28)$ revealed both supportive and contradictory outcomes with SP sports and the NEH. Therefore, the NEH is a dynamic and situational concept that depends on several agents including movement characteristics (e.g., side of the movement), hemisphere, and athletes' traits.

More generally, neural efficiency is analogous to driving the same route to/from work for several years. You become progressively familiar with the route, enabling you to drive faster and with lower gas consumption compared to your first time driving on these roads. However, this efficiency is situational, depending on other contextual factors such as the traffic, weather, and other states that may fluctuate from one day to the next (e.g., your mood, alertness, etc.). Therefore, neuroplasticity is 
ever-present in human life, and its characteristics are individualand situation-dependent (Gourgouvelis et al., 2017; Berti et al., 2019).

\section{SUMMARY AND FUTURE DIRECTIONS}

In this study, selection, information, and analysis meta-biases were controlled rigorously by following the pre-established systematic review methods (Page et al., 2021). Studies on the NEH of intelligence (Haier et al., 1988; Neubauer et al., 2002) have found that expert individuals have lower brain activity when performing cognitive tasks because they tend to activate only the brain areas that are needed to complete the task. This has been explained as resulting from a genetic predisposition combined with extensive training (Callan and Naito, 2014). We demonstrated that several previous studies supported this theory, although subsequent research uncovered conflicting data, a paradox, or found pathways that moderate the perceptualcognitive brain activation relationship (Alves et al., 2013). In this way, neural efficiency may indeed act as a factor for expertnovice brain region activation distinction as the various sports involved in 28 studies. Moreover, neural performance appears to be related to the amount and quality of training received. We examined the neuroscientific evidence from those studies, which revealed that long-term specific training might improve athletes' top-down processing pathway connectivity (Oliver et al., 2020). It is beneficial to the effect of unconscious resources in the frontal region on motor processing in the early stage, which improves the efficiency in fast-task response performance. This change could save limited attention resources for succeeding activation in motor task processing. More specifically, the visual $\mathrm{N} 1$ component induced by frontal and central regions were associated with the early reaction preparation processing (Vogel and Luck, 2000). The higher amplitude of N1 provoked by sports in the parietal-central area indicated that athletes consume more attention resources in the early stage of behavioral response, which may be related to the long-term specialized training. In one study (17), athletes can respond more quickly through early attention processing. The amplitude of $\mathrm{N} 1$ induced by athletes' frontal region was higher than that of the control group. Accordingly, experts have the capacity to conduct fast and accurate movement to satisfy the need for rapid action response during performance, saving attention resources for late brain executive processing, and promoting the cerebral cortex neural efficiency.

From integrating the aforementioned evidence, we concluded that the neural efficiency phenomenon is most commonly observed for frontal brain areas when athletes are confronted with spatial and perceptual-cognitive tasks of low to moderate task complexity (Neubauer and Fink, 2009; Alves et al., 2013). Compared with the initial training, the activation of subjects' frontal-parietal cortex gradually decreased with the proficiency of the skills, which theoretically supported the NEH (Haier et al., 1992; Grabner et al., 2006; Bueichekú et al., 2016). However, in highly complex tasks, both experts and novices seem to be able to stimulate more cortical resources, thus from the perspective of proficiency in brain function, there is a positive correlation between brain use and cognitive ability (Neubauer and Fink, 2009). Therefore, the inconsistencies in selected studies suggests that there may be conditional limits on the neural efficiency of the cerebral cortex in athletes.

According to the current evidence derived from selected studies, the degree of effort, as well as the cognitive demands of the control systems involved in the task, will influence individuals' efficient and effortful processing during performance $(4,6-8,10,15,17-19,24,28)$. Athletes could benefit from transcranial direct current stimulation of targeted brain areas to improve learning through enhanced long-term potentiation while performing the task (Coffman et al., 2014; Flöel, 2014; Prichard et al., 2014). However, it is possible that this reversal of brain activation-professional training relationship is caused by athletes' psychomotor decision to motivate more commitment as compared to the novices, and the novices actually have insufficient training that the task surpasses their neural efficacy $(9,10,22)$. In this way, as a result of recent neuromodulation findings and neuroimage methods, there are four potential future directions that may assist to clarify the detailed mechanism of neural efficiency: neuroimage technologies, study design, interdisciplinary research, and heterogenous populations.

Neuroimaging technologies are mutually complementary. Synchronization of high frequency rhythmic waves (i.e., gamma), in the occipital cortex of the subjects indicated that the task induced the occipital cortex. Previous studies $(23,25)$ have confirmed that high-frequency rhythmic wave oscillation is closely related to cognitive processing. Further analysis of gamma and alpha rhythms is considered in the later stage oscillation characteristics will be possible to better reveal the mechanism of athletes' brain neural efficiency. Meanwhile, the synchronization of neural clusters in the brain regions reflects the information processing characteristics of the brain neural network. Therefore, future studies should consider the degree of connection between the activation of brain regions and the function of brain regions and reveal the neural processing characteristics of the athletes' brains and the physiological mechanism of the neural efficiency of motor mobilization from the perspective of the whole brain network. In addition, the lack of spatial resolution of EEG also restricts the accurate localization of oscillating signal elicitors. Therefore, as Study 11 conducted, it is necessary to consider the combination of EEG and fMRI technologies to further explore the neural efficiency of athletes' brains.

Further, more rigorous designs are encouraged in future NEH experiments. Several studies (e.g., 22, 24) used simplemoderate recognition tasks that were familiar to all subjects, ensuring the ecological validity of the study and inducing the same cortical pathway to improve the comparability of cortical neural efficiency among the subjects. However, using simple-moderate general tasks cannot guarantee the specificity of special task processing. Studies (e.g., 18, 23, 25) used moderatecomplex specific motor tasks could not eliminate the influence of knowledge factors such as action recognition strategy on cortex activation and functional connection (Poldrack, 2015). Therefore, it is necessary to optimize the design of experimental tasks to ensure that the same cortical circuits are induced 
while avoiding the interference effect of experiential knowledge on the differentiation of neural cortex functions. Moreover, the neural efficiency of an athlete's brain is a reflection of the microcosmic functional mechanism of cerebral cortex neurons, which non-traumatic neuroimaging studies cannot comprehensively investigate (Kandel et al., 2000). Therefore, interdisciplinary research is promising as a complementary approach to further explore the NEH at the molecular level, such as using animal models.

Lastly, broader populations and tasks will likely reveal more complementary evidence. According to Wang et al. (2013), tennis players have better inhibitory control than swimmers and novices, implying that training in open skill sports might promote fundamental cognitive control. While the $\mathrm{NEH}$ has recently been applied to a number of moment-related tasks, it is unknown whether neural efficiency exists in the broader population and different type of sports (e.g., long term specific training in individual and team sports, or adults and under 18 population). Moreover, we notice relative research revealed substantial EEG and MRI data which is encouraging but not convenient for researchers to compare outcomes parallelly. In this way, although the heterogeneous outcomes might be one of the barriers, adding more meta-analysis to the field is encouraging because a single summary estimate is more rigorous than systematic reviews and offers quantitative evidence of the long-term training effect on athletes' brains. Of note, in the future, pronounced evidence of the $\mathrm{NEH}$ also might be beneficial to patients with brain damage or related disease (e.g., Alzheimer's, Autism, Attention Deficit Hyperactivity Disorder)

\section{REFERENCES}

Alves, H., Voss, M. W., Boot, W. R., Deslandes, A., Cossich, V., Salles, J. I., et al. (2013). Perceptual-cognitive expertise in elite volleyball players. Front. Psychol. 4:36. doi: 10.3389/fpsyg.2013.00036

Babiloni, C., Del Percio, C., Rossini, P. M., Marzano, N., Iacoboni, M., Infarinato, F., et al. (2009). Judgment of actions in experts: a high-resolution EEG study in elite athletes. Neuroimage 45, 512-521. doi: 10.1016/j.neuroimage.2008. 11.035

Babiloni, C., Marano, N., Infarinato, F., Iacoboni, M., Rizza, G., Aschieri, P., et al. (2010). "Neural efficiency" of experts' brain during judgment of actions: a highresolution EEG study in elite and amateur karate athletes. Behav. Brain Res. 207, 466-475. doi: 10.1016/j.bbr.2009.10.034

Baker, J., Wattie, N., and Schorer, J. (2015). "Defining expertise: a taxonomy for researchers in skill acquisition and expertise," in Routledge Handbook of Sport Expertise, 1st Edn, ed J. Baker and D. Farrow (New York, NY: Routledge), $145-155$.

Berti, B., Momi, D., Sprugnoli, G., Neri, F., Bonifazi, M., Rossi, A., et al. (2019). Peculiarities of functional connectivity-including cross-modal patterns in professional karate athletes: Correlations with cognitive and motor performances. Neural Plast. 2019:6807978. doi: 10.1155/2019/68 07978

Bertollo, M., di Fronso, S., Conforto, S., Schmid, M., Bortoli, L., Comani, S., et al. (2016). Proficient brain for optimal performance: the MAP model perspective. PeerJ 4:e2082. doi: 10.7717/peerj.2082

Bueichekú, E., Miró-Padilla, A., Palomar-García, M. Á., Ventura-Campos, N., Parcet, M. A., Barrós-Loscertales, A., et al. (2016). Reduced posterior parietal cortex activation after training on a visual search task. Neuroimage 135, 204-213. doi: 10.1016/j.neuroimage.2016.04.059 in the field of neurorehabilitation. Appropriate dose of exercise intensity, duration, and frequency may be useful in efforts to improve FC and reduce irrelevant information processing in a long-term perspective. In conclusion, this study has provided a comprehensive review of studies of neural efficiency distinguishing expert and novice athletes, raising several issues and directions for future research.

\section{DATA AVAILABILITY STATEMENT}

The original contributions presented in the study are included in the article/Supplementary Material, further inquiries can be directed to the corresponding author/s.

\section{AUTHOR CONTRIBUTIONS}

LL and DS contributed to the conception and design of the study. LL wrote the first draft of the manuscript and critically revising the draft for important intellectual content. DS contributed to revising and approving the final version of the manuscript. All authors contributed to the article and approved the submitted version.

\section{SUPPLEMENTARY MATERIAL}

The Supplementary Material for this article can be found online at: https://www.frontiersin.org/articles/10.3389/fnbeh. 2021.698555/full\#supplementary-material
Callan, D. E., and Naito, E. (2014). Neural processes distinguishing elite from expert and novice athletes. Cogn. Behav. Neurol. 27, 183-188. doi: 10.1097/WNN.0000000000000043

Calvo-Merino, B., Glaser, D. E., Grèzes, J., Passingham, R. E., and Haggard, P. (2005). Action observation and acquired motor skills: an FMRI study with expert dancers. Cerebral Cortex 15, 1243-1249. doi: 10.1093/cercor/bhi007

Coffman, B. A., Clark, V. P., and Parasuraman, R. (2014). Battery powered thought: enhancement of attention, learning, and memory in healthy adults using transcranial direct current stimulation. Neuroimage 85, 895-908. doi: 10.1016/j.neuroimage.2013.07.083

Cook, R., Bird, G., Catmur, C., Press, C., and Heyes, C. (2014). Mirror neurons: from origin to function. Behav. Brain Sci. 37, 177-192. doi: 10.1017/S0140525X13000903

Costanzo, M. E., VanMeter, J. W., Janelle, C. M., Braun, A., Miller, M. W., Oldham, J., et al. (2016). Neural efficiency in expert cognitivemotor performers during affective challenge. J. Mot. Behav. 48, 573-588. doi: 10.1080/00222895.2016.1161591

Del Percio, C., Babiloni, C., Bertollo, M., Marzano, N., Iacoboni, M., Infarinato, F., et al. (2009a). Visuo-attentional and sensorimotor alpha rhythms are related to visuo-motor performance in athletes. Hum. Brain Mapp. 30, 3527-3540. doi: 10.1002/hbm.20776

Del Percio, C., Babiloni, C., Marzano, N., Iacoboni, M., Infarinato, F., Vecchio, F., et al. (2009b). "Neural efficiency" of athletes' brain for upright standing: a high-resolution EEG study. Brain Res. Bull. 79, 193-200. doi: 10.1016/j.brainresbull.2009.02.001

Del Percio, C., Franzetti, M., De Matti, A. J., Noce, G., Lizio, R., Lopez, S., et al. (2019). Football players do not show "neural efficiency" in cortical activity related to visuospatial information processing during football scenes: an EEG mapping study. Front. Psychol. 10:890. doi: 10.3389/fpsyg.2019.00890 
Del Percio, C., Infarinato, F., Iacoboni, M., Marzano, N., Soricelli, A., Aschieri, P., et al. (2010). Movement-related desynchronization of alpha rhythms is lower in athletes than non-athletes: a high-resolution EEG study. Clin. Neurophysiol. 121, 482-491. doi: 10.1016/j.clinph.2009.12.004

Del Percio, C., Infarinato, F., Marzano, N., Iacoboni, M., Aschieri, P., Lizio, R., et al. (2011). Reactivity of alpha rhythms to eyes opening is lower in athletes than non-athletes: a high-resolution EEG study. Int. J. Psychophysiol. 82, 240-247. doi: 10.1016/j.ijpsycho.2011.09.005

Del Percio, C., Rossini, P. M., Marzano, N., Iacoboni, M., Infarinato, F., Aschieri, P., et al. (2008). Is there a "neural efficiency" in athletes? A high-resolution EEG study. Neuroimage 42, 1544-1553. doi: 10.1016/j.neuroimage.2008. 05.061

Di Fronso, S., Robazza, C., Filho, E., Bortoli, L., Comani, S., and Bertollo, M. (2016). Neural markers of performance states in an Olympic athlete: an EEG case study in air-pistol shooting. J. Sports Sci. Med. 15, 214-222.

Di Russo, F., Pitzalis, S., Aprile, T., and Spinelli, D. (2005). Effect of practice on brain activity: an investigation in top-level rifle shooters. Med. Sci. Sports Exerc. 37:1586. doi: 10.1249/01.mss.0000177458.71676.0d

Dunst, B., Benedek, M., Jauk, E., Bergner, S., Koschutnig, K., Sommer, M., et al. (2014). Neural efficiency as a function of task demands. Intelligence 42, 22-30. doi: 10.1016/j.intell.2013.09.005

Duru, A. D., and Assem, M. (2018). Investigating neural efficiency of elite karate athletes during a mental arithmetic task using EEG. Cogn. Neurodyn. 12, 95-102. doi: 10.1007/s11571-017-9464-y

Eng, H. Y., Chen, D., and Jiang, Y. (2005). Visual working memory for simple and complex visual stimuli. Psychon. Bull. Rev. 12, 1127-1133. doi: 10.3758/BF03206454

Eslinger, P. J., and Tranel, D. (2005). Integrative study of cognitive, social, and emotional processes in clinical neuroscience. Cogn. Behav. Neurol. 18, 1-4. doi: 10.1097/01.wnn.0000152206.73195.1e

Fargier, P., Collet, C., Moran, A., and Massarelli, R. (2017). Inter-disciplinarity in sport sciences: the neuroscience example. Eur. J. Sport Sci. 17, 42-50. doi: 10.1080/17461391.2016.1207710

Fattapposta, F., Amabile, G., Cordischi, M. V., Di Venanzio, D., Foti, A., Pierelli, F., et al. (1996). Long-term practice effects on a new skilled motor learning: an electrophysiological study. Electroencephalogr Clin Neurophysiol. 99, 495-507. doi: 10.1016/S0013-4694(96)96560-8

Filho, E., Dobersek, U., and Husselman, T. A. (2021). The role of neural efficiency, transient hypofrontality and neural proficiency in optimal performance in self-paced sports: a meta-analytic review. Exp. Brain Res. 239, 1381-1393. doi: 10.1007/s00221-021-06078-9

Fleddermann, M. T., Heppe, H., and Zentgraf, K. (2019). Off-court generic perceptual-cognitive training in elite volleyball athletes: task-specific effects and levels of transfer. Front. Psychol. 10:1599. doi: 10.3389/fpsyg.2019. 01599

Flöel, A. (2014). tDCS-enhanced motor and cognitive function in neurological diseases. Neuroimage 85, 934-947. doi: 10.1016/j.neuroimage.2013.05.098

Gevins, A., and Smith, M. E. (2000). Neurophysiological measures of working memory and individual differences in cognitive ability and cognitive style. Cerebral Cortex 10, 829-839. doi: 10.1093/cercor/10.9.829

Gourgouvelis, J., Yielder, P., and Murphy, B. (2017). Exercise promotes neuroplasticity in both healthy and depressed brains: an fMRI pilot study. Neural Plast. 2017, 1687-5443. doi: 10.1155/2017/8305287

Grabner, R. H., Neubauer, A. C., and Stern, E. (2006). Superior performance and neural efficiency: the impact of intelligence and expertise. Brain Res. Bull. 69, 422-439. doi: 10.1016/j.brainresbull.2006.02.009

Granacher, U., and Borde, R. (2017). Effects of sport-specific training during the early stages of long-term athlete development on physical fitness, body composition, cognitive, and academic performances. Front. Physiol. 8:810. doi: 10.3389/fphys.2017.00810

Guo, Z., Li, A., and Yu, L. (2017). "Neural efficiency" of athletes' brain during visuo spatial task: an fMRI study on table tennis players. Front. Behav. Neurosci. 11, 1-8. doi: 10.3389/fnbeh.2017.00072

Haier, R. J., Siegel Jr, B. V., MacLachlan, A., Soderling, E., Lottenberg, S., and Buchsbaum, M. S. (1992). Regional glucose metabolic changes after learning a complex visuospatial/motor task: a positron emission tomographic study. Brain Res. 570, 134-143. doi: 10.1016/0006-8993(92)90573-R
Haier, R. J., Siegel Jr, B. V., Nuechterlein, K. H., Hazlett, E., Wu, J. C., Paek, J., et al. (1988). Cortical glucose metabolic rate correlates of abstract reasoning and attention studied with positron emission tomography. Intelligence 12, 199-217. doi: 10.1016/0160-2896(88)90016-5

Hatta, A., Nishihira, Y., Higashiura, T., Kim, S. R., and Kaneda, T. (2009). Longterm motor practice induces practice-dependent modulation of movementrelated cortical potentials (MRCP) preceding a self-paced non-dominant handgrip movement in kendo players. Neurosci. Lett. 459, 105-108. doi: 10.1016/j.neulet.2009.05.005

Haufler, A. J., Spalding, T. W., Santa Maria, D. L., and Hatfield, B. D. (2000). Neuro-cognitive activity during a self-paced visuospatial task: comparative EEG profiles in marksmen and novice shooters. Biol. Psychol. 53, 131-160. doi: 10.1016/S0301-0511(00)00047-8

Hwang, R. J., Chen, H. J., Guo, Z. X., Lee, Y. S., and Liu, T. Y. (2019). Effects of aerobic exercise on sad emotion regulation in young women: an electroencephalograph study. Cogn. Neurodyn. 13, 33-43. doi: 10.1007/s11571-018-9511-3

Iwadate, M., Mori, A., Ashizuka, T., Takayose, M., and Ozawa, T. (2005). Longterm physical exercise and somatosensory event-related potentials. Exp. Brain Res. 160, 528-532. doi: 10.1007/s00221-004-2125-5

Janelle, C. M., Hillman, C. H., Apparies, R. J., Murray, N. P., Meili, L., Fallon, E. A., et al. (2000). Expertise differences in cortical activation and gaze behavior during rifle shooting. J. Sport Exercise Psychol. 22, 167-182. doi: 10.1123 /jsep.22.2.167

Kandel, E. R., Schwartz, J. H., Jessell, T. M., Siegelbaum, S., Hudspeth, A. J., and Mack, S. (2000). Principles of Neural Science. New York, NY: McGraw-Hill.

Kim, W., Chang, Y., Kim, J., Seo, J., Ryu, K., Lee, E., et al. (2014). An fMRI study of differences in brain activity among elite, expert, and novice archers at the moment of optimal aiming. Cogn. Behav. Neurol. 27, 173-182. doi: 10.1097/WNN.0000000000000042

Kita, Y., Mori, A., and Nara, M. (2001). Two types of movement-related cortical potentials preceding wrist extension in humans. Neuroreport 12, 2221-2225. doi: 10.1097/00001756-200107200-00035

Kliger, L., and Yovel, G. (2020). The functional organization of high-level visual cortex determines the representation of complex visual stimuli. J. Neurosci. 40, 7545-7558. doi: 10.1523/JNEUROSCI.0446-20.2020

Loze, G. M., Collins, D., and Holmes, P. S. (2001). Pre-shot EEG alpha-power reactivity during expert air-pistol shooting: a comparison of best and worst shots. J. Sports Sci. 19, 727-733. doi: 10.1080/02640410152475856

Mann, D. T., Wright, A., and Janelle, C. M. (2016). Quiet eye: the efficiency paradox comment on Vickers. Curr. Issues Sport Sci. 1:e000858. doi: 10.15203/CISS_2016.111

Milton, J., Solodkin, A., Hluštík, P., and Small, S. L. (2007). The mind of expert motor performance is cool and focused. Neuroimage 35, 804-813. doi: 10.1016/j.neuroimage.2007.01.003

Naito, E., and Hirose, S. (2014). Efficient foot motor control by Neymar's brain. Front. Hum. Neurosci. 8, 1-7. doi: 10.3389/fnhum.2014.00594

Nakamoto, H., and Mori, S. (2008). Effects of stimulus-response compatibility in mediating expert performance in baseball players. Brain Res. 1189, 179-188. doi: 10.1016/j.brainres.2007.10.096

Nakata, H. (2020). The athletes' brain. Adv. Exercise Sports Physiol. 26, 31-34. Available online at: https://jglobal.jst.go.jp/en/detail?JGLOBAL_ID= 202102264157747241

Neubauer, A. C., and Fink, A. (2009). Intelligence and neural efficiency. Neurosci. Biobehav. Rev. 33, 1004-1023. doi: 10.1016/j.neubiorev.2009. 04.001

Neubauer, A. C., Fink, A., and Schrausser, D. G. (2002). Intelligence and neural efficiency: the influence of task content and sex on the brain-IQ relationship. Intelligence 30, 515-536. doi: 10.1016/S0160-2896(02)00091-0

Neubauer, A. C., Grabner, R. H., Freudenthaler, H. H., Beckmann, J. F., and Guthke, J. (2004). Intelligence and individual differences in becoming neurally efficient. Acta Psychol. 116, 55-74. doi: 10.1016/j.actpsy.2003.11.005

Nishiguchi, S., Yamada, M., Tanigawa, T., Sekiyama, K., Kawagoe, T., Suzuki, M., et al. (2015). A 12-week physical and cognitive exercise program can improve cognitive function and neural efficiency in community-dwelling older adults: a randomized controlled trial. J. Am. Geriatr. Soc. 63, 1355-1363. doi: 10.1111 /jgs.13481 
Oliver, A., McCarthy, P. J., and Burns, L. (2020). Teaching athletes to understand their attention is teaching them to concentrate. J. Sport Psychol. Act. 1-15. doi: 10.1080/21520704.2020.1838980

Olsson, C. J., Jonsson, B., Larsson, A., and Nyberg, L. (2008). Motor representations and practice affect brain systems underlying imagery: an fMRI study of internal imagery in novices and active high jumpers. Open Neuroimag. J. 2, 5-13. doi: 10.2174/1874440000802010005

Page, M. J., Moher, D., Bossuyt, P. M., Boutron, I., Hoffmann, T. C., Mulrow, C. D., et al. (2021). PRISMA 2020 explanation and elaboration: updated guidance and exemplars for reporting systematic reviews. Br. Med. J. 372:n160. doi: 10.1136/bmj.n160

Paolicelli, R. C., Bolasco, G., Pagani, F., Maggi, L., Scianni, M., Panzanelli, P., et al. (2011). Synaptic pruning by microglia is necessary for normal brain development. Science 333, 1456-1458. doi: 10.1126/science.1202529

Papousek, I., and Schulter, G. (2004). Manipulation of frontal brain asymmetry by cognitive tasks. Brain Cogn. 54, 43-51. doi: 10.1016/S0278-2626(03)00258-6

Park, I., Kim, Y., and Kim, S. K. (2020). Athlete-specific neural strategies under pressure: a fnirs pilot study. Int. J. Environ. Res. Public Health 17, 1-12. doi: 10.3390/ijerph17228464

Park, J. L., Fairweather, M. M., and Donaldson, D. I. (2015). Making the case for mobile cognition: EEG and sports performance. Neurosci. Biobehav. Rev. 52, 117-130. doi: 10.1016/j.neubiorev.2015.02.014

Parr, J. V. V., Gallicchio, G., Harrison, N. R., Johnen, A. K., and Wood, G. (2020). All talk? Challenging the use of left-temporal EEG alpha oscillations as valid measures of verbal processing and conscious motor control. Biol. Psychol. 155:107943. doi: 10.1016/j.biopsycho.2020.107943

Poldrack, R. A. (2015). Is "efficiency" a useful concept in cognitive neuroscience? Dev. Cogn. Neurosci. 11, 12-17. doi: 10.1016/j.dcn.2014. 06.001

Prichard, G., Weiller, C., Fritsch, B., and Reis, J. (2014). Effects of different electrical brain stimulation protocols on subcomponents of motor skill learning. Brain Stimul. 7, 532-540. doi: 10.1016/j.brs.2014.04.005

Qiu, F., Pi, Y., Liu, K., Zhu, H., Li, X., Zhang, J., et al. (2019). Neural efficiency in basketball players is associated with bidirectional reductions in cortical activation and deactivation during multiple object tracking task performance. Biol. Psychol. 144, 28-36. doi: 10.1016/j.biopsycho.2019.03.008

Rypma, B., and Prabhakaran, V. (2009). When less is more and when more is more: the mediating roles of capacity and speed in brain-behavior efficiency. Intelligence 37, 207-222. doi: 10.1016/j.intell.2008.12.004

Schmidt, R. A., and Lee, T. D. (2014). Motor Learning and Performance: From Principles to Application. 5th Edn. Champaign, IL: Human Kinetics.

Shepherd, J. (2015). Conscious control over action. Mind Lang. 30, 320-344. doi: $10.1111 /$ mila. 12082

Singer, R. N. (2000). Performance and human factors: considerations about cognition and attention for self-paced and externally-paced events. Ergonomics 43, 1661-1680. doi: 10.1080/001401300750004078

Smith, D. M. (2016). Neurophysiology of action anticipation in athletes: a systematic review. Neurosci. Biobehav. Rev. 60, 115-120. doi: 10.1016/j.neubiorev.2015.11.007

Szucs, D., and Ioannidis, J. P. (2020). Sample size evolution in neuroimaging research: an evaluation of highly-cited studies (1990-2012) and of latest practices (2017-2018) in high-impact journals. Neuroimage 221:117164. doi: 10.1016/j.neuroimage.2020.117164

Turella, L., Wurm, M. F., Tucciarelli, R., and Lingnau, A. (2013). Expertise in action observation: recent neuroimaging findings and future perspectives. Front. Hum. Neurosci. 7:637. doi: 10.3389/fnhum.2013.00637

Van Eck, N. J., and Waltman, L. (2010). Software survey: VOSviewer, a computer program for bibliometric mapping. Scientometrics 84, 523-538. doi: 10.1007/s11192-009-0146-3
Vickers, J. N. (2016). The quiet eye: Origins, controversies, and future directions. Kinesiol. Rev. 5, 119-128. doi: 10.1123/kr.2016-0005

Vickers, J. N., and Williams, A. M. (2017). "The role of mental processes in elite sports performance," in Oxford Research Encyclopedia of Psychology. doi: 10.1093/acrefore/9780190236557.013.161

Vogel, E. K., and Luck, S. J. (2000). The visual N1 component as an index of a discrimination process. Psychophysiology 37, 190-203. doi: 10.1111/1469-8986.3720190

Wang, C. H., Chang, C. C., Liang, Y. M., Shih, C. M., Chiu, W. S., Tseng, P., et al. (2013). Open vs. closed skill sports and the modulation of inhibitory control. PLoS ONE 8:e55773. doi: 10.1371/journal.pone.0055773

Wang, C. H., and Tu, K. C. (2017). Neural correlates of expert behavior during a domain-specific attentional cueing task in badminton players. J. Sport Exercise Psychol. 39, 209-221. doi: 10.1123/jsep.2016-0335

Wei, Y., and Li, A. (2017). Neural efficiency of athletes cerebral cortex: the evidence of ERP and ERD/S during simple visual discrimination task. J. Tianjin Univ. Sport 32, 434-442. doi: 10.13297/j.cnki.issn1005-0000.2017. 05.010

Wei, Y., and Li, A. (2018). Neural efficiency of athletes' specific motor recognition: the evidence from EEG rhythm desynchronization and coherence. J. Tianjin Univ. Sport 33, 311-320. doi: 10.13297/j.cnki.issn1005-0000.2018.04.006

Wolf, S., Brölz, E., Scholz, D., Ramos-Murguialday, A., Keune, P. M., Hautzinger, M., et al. (2014). Winning the game: brain processes in expert, young elite and amateur table tennis players. Front. Behav. Neurosci. 8:370. doi: $10.3389 /$ fnbeh. 2014.00370

Yamashiro, K., Sato, D., Onishi, H., and Maruyama, A. (2018). Plastic changes in an athlete's brain. Adv. Exercise Sports Physiol. 24, 1-5. Available online at: https:// id.ndl.go.jp/bib/029116250

Yamashiro, K., Sato, D., Onishi, H., Sugawara, K., Nakazawa, S., Shimojo, H., et al. (2015). Skill-specific changes in somatosensory nogo potentials in baseball players. PLoS ONE 10:e0142581. doi: 10.1371/journal.pone.0142581

Yang, C., Luo, N., Liang, M., Zhou, S., Yu, Q., and Zhang, J. (2020). Altered brain functional connectivity density in fast-ball sports athletes with early stage of motor training. Front. Psychol. 11:530122. doi: 10.3389/fpsyg.2020.5 30122

Zamora-López, G., Zhou, C., and Kurths, J. (2010). Cortical hubs form a module for multisensory integration on top of the hierarchy of cortical networks. Front. Neuroinform. 4:1. doi: 10.3389/neuro.11.001.2010

Zhang, L., Qiu, F., Zhu, H., Xiang, M., and Zhou, L. (2019). Neural efficiency and acquired motor skills: an fMRI study of expert athletes. Front. Psychol. 10:2752. doi: 10.3389/fpsyg.2019.02752

Conflict of Interest: The authors declare that the research was conducted in the absence of any commercial or financial relationships that could be construed as a potential conflict of interest.

Publisher's Note: All claims expressed in this article are solely those of the authors and do not necessarily represent those of their affiliated organizations, or those of the publisher, the editors and the reviewers. Any product that may be evaluated in this article, or claim that may be made by its manufacturer, is not guaranteed or endorsed by the publisher.

Copyright (C) $2021 \mathrm{Li}$ and Smith. This is an open-access article distributed under the terms of the Creative Commons Attribution License (CC BY). The use, distribution or reproduction in other forums is permitted, provided the original author(s) and the copyright owner(s) are credited and that the original publication in this journal is cited, in accordance with accepted academic practice. No use, distribution or reproduction is permitted which does not comply with these terms. 